\title{
Practical Techniques for Modeling Gas Turbine Engine Performance
}

\author{
Jeffryes W. Chapman* \\ Vantage Partners LLC., Brook Park OH, 44142, USA \\ Thomas M. Lavelle ${ }^{\dagger}$ and Jonathan S. Litt ${ }^{\ddagger}$ \\ NASA Glenn Research Center, Cleveland OH, 44135, USA
}

\begin{abstract}
The cost and risk associated with the design and operation of gas turbine engine systems has led to an increasing dependence on mathematical models. In this paper, the fundamentals of engine simulation will be reviewed, an example performance analysis will be performed, and relationships useful for engine control system development will be highlighted. The focus will be on thermodynamic modeling utilizing techniques common in industry, such as: the Brayton cycle, component performance maps, map scaling, and design point criteria generation. In general, these topics will be viewed from the standpoint of an example turbojet engine model; however, demonstrated concepts may be adapted to other gas turbine systems, such as gas generators, marine engines, or high bypass aircraft engines. The purpose of this paper is to provide an example of gas turbine model generation and system performance analysis for educational uses, such as curriculum creation or student reference.
\end{abstract}

\section{Nomenclature}

Alt $\quad$ Altitude

$A_{t h} \quad$ Throat area

CFD Computational Fluid Dynamics

$C_{D} \quad$ Coefficient of drag

$C_{f g} \quad$ Coefficient of thrust

$C_{p} \quad$ Specific heat at constant pressure

$C_{v} \quad$ Specific heat at constant volume

$d P \quad$ Change in pressure

$d T \quad$ Temperature deviation from standard day (518.67 $R$ at sea level static conditions)

Eff Efficiency

$E P R \quad$ Engine pressure ratio

$F \quad$ Thrust, $l b f$

$F_{d} \quad$ Drag, $l b f$

$F_{g} \quad$ Gross thrust, $l b f$

$F_{n} \quad$ Net thrust, $l b f$

$F A R \quad$ Fuel to air ratio

$g \quad$ Gravity constant, $32.17 \frac{f t \cdot l b m}{l b f \cdot s^{2}}$

$h \quad$ Enthalpy, $\frac{B T U}{l b m}$

hs Static enthalpy, $\frac{B T U}{B l b m}$

ht Total enthalpy, $\frac{B T U}{l b m}$

$h 2 t \quad$ Enthalpy to temperature table lookup

I Inertia, slug $\cdot f t^{2}$

\footnotetext{
*Research Engineer, 3000 Aerospace Parkway, Brook Park OH, AIAA Member.

${ }^{\dagger}$ Research Engineer, 21000 Brookpark Rd., Cleveland OH, MS 5-11, AIAA Member.

${ }^{\ddagger}$ Research Engineer, 21000 Brookpark Rd., Cleveland OH, MS 77-1, AIAA Senior Member.
} 


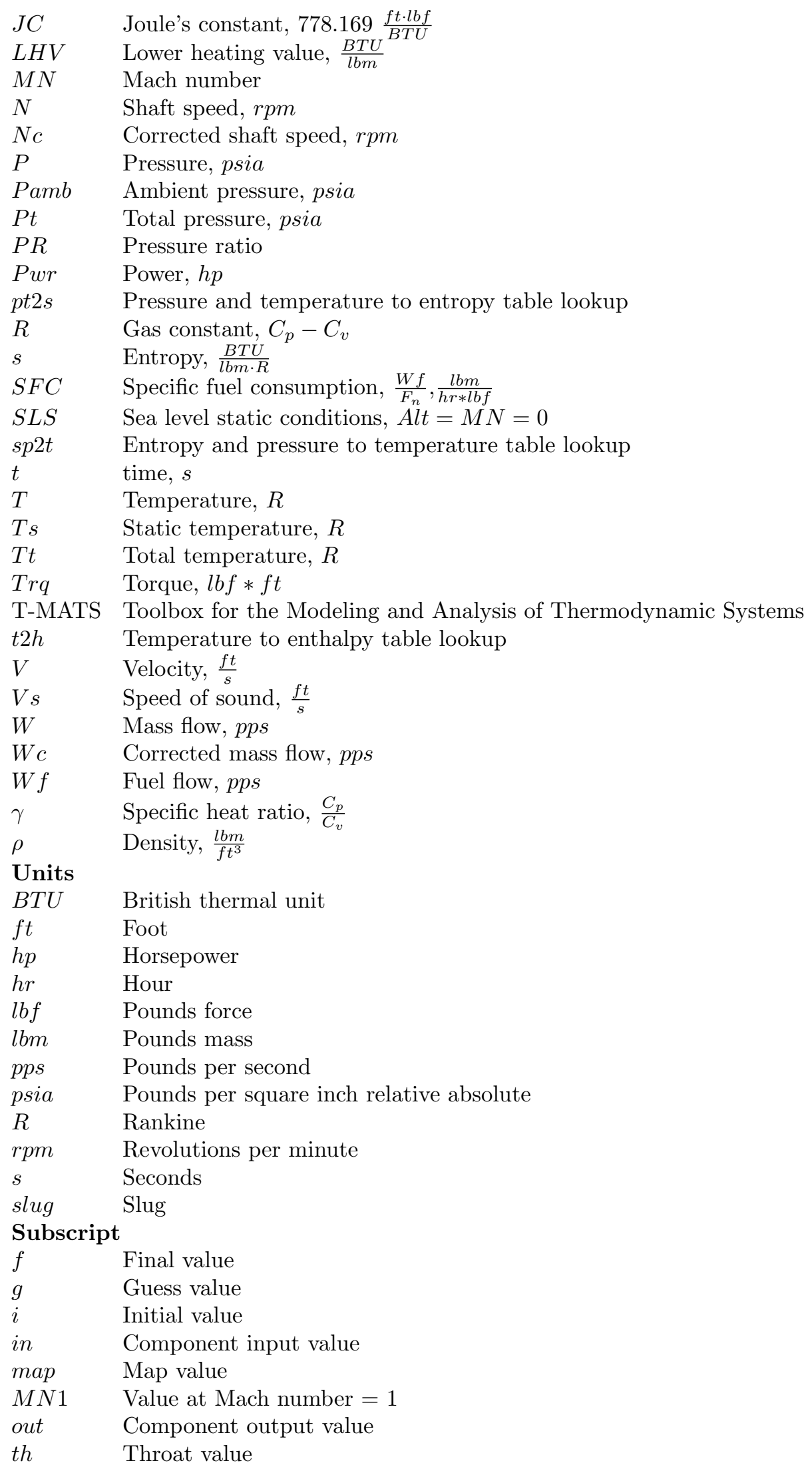




\section{Introduction}

Gas turbine engines play an integral part in the modern world and are commonly utilized in applications such as generating electricity or powering vehicles like airplanes, helicopters, and watercraft. As these machines have become more advanced and complex, the cost of hardware testing has risen. Because of this, it is no surprise that gas turbine simulations have become an invaluable tool at all phases of the engine life cycle.

In general, a commercial aircraft engine consists of a compressor, burner, turbine, and nozzle, in series. The compressor and turbine add power to or harness power from the gas stream, or mass flow, through the rotation of specialized blades. Power generated by mass flowing through the turbine can be used to rotate one or more shafts connected to the compressor or other rotating machinery. ${ }^{1}$ Engine thrust is generated by air moving out of the back of the engine. The amount of thrust is mainly determined by the amount of air and its velocity. The nozzle component acts as a mechanism to maximize thrust by converting excess pressure from the engine exhaust or bypass into air velocity.

Analysis of gas turbines typically comes in two distinct types, parametric cycle and performance. ${ }^{2}$ Parametric cycle, or design point, analysis is used by developers to perform the engine cycle design (design of the engine system's overall thermodynamic cycle). This process utilizes component design choices to meet performance requirements, while taking into account system limitations and flight conditions. Essentially, cycle design uses operational requirements to size the various engine components at key mission points. As a simplified example, a designer may be required to create an engine by altering the compressor pressure ratio and bypass ratio to maintain 30,000 lbf of thrust at take off conditions and 1,000 lbf thrust with 0.9 specific fuel consumption $(S F C)$ at a cruise altitude of 30,000 ft. Performance, or off-design, analysis is an examination of the overall operational envelope. This analysis assumes all component design decisions have been completed and can be used to predict performance not explicitly defined during the cycle design, verifying operation within the system constraints.

Gas turbine modeling in this paper uses a combination of empirical and physics-based techniques. Modeling equations maintain a thermodynamic cycle approach based on the Brayton cycle, and ensure conservation of energy and momentum across the system. In the Brayton cycle, compression and expansion are isentropic and adiabatic, and combustion occurs at a constant pressure. Compressor and turbine operation is simulated with performance maps. These maps are non-linear empirical component models that can be generated with computational fluid dynamics (CFD), experimental data, or estimated using generic maps. The use of pregenerated generic maps, scaled to the application, can be a convenient way to obtain models for unknown turbomachinery. While these scaled maps may enable satisfactory matching for an application near the design point, significant error can accumulate as the engine is moved around the operational envelope.

This paper will mainly discuss three engine simulation topics: component level modeling, system level modeling using limited data sets, and performance analysis. These topics will be explored through the development and analysis of an example turbojet model. In this exercise, design point analysis will be reviewed only for the purposes of matching data for modeling, and cycle design will not be discussed. The performance analysis will be conducted for the purposes of detailing the parameters of interest at all operational points between idle and full power. This analysis will cover the relationships between thrust, SFC, engine mass flow, shaft speed, engine pressure ratios, Mach number $(M N)$, and altitude for the purposes of predicting performance and engine control. During engine operation, it is imperative that certain limits are not exceeded, which allows the engine to maintain safe operation. In general, these limits are quantified based on measurable parameters such as speeds, temperatures, and pressures. Performance modeling will be used to analyze engine operation outside the typical design points to gain an understanding of engine operation and verify these safety margins are met. To keep the process simple, this paper will consider steady-state limits and engine operation. While this is acceptable for a first cut estimation of system performance, a full dynamic analysis of the engine with a control system must be performed to verify the engine performs as required during transients.

Techniques in this paper are demonstrated using the Toolbox for the Modeling and Analysis of Thermodynamic Systems (T-MATS), ${ }^{3}$ which utilizes MATLAB ${ }^{\circledR} /$ Simulink $^{\circledR}$, a dynamic simulation software package, and Cantera, ${ }^{4}$ a high fidelity thermodynamic process software. Free and open source, T-MATS packages thermodynamic components into a framework that enables easy creation of complex systems and contains everything required for the generation of gas turbine models. Previous work has documented that T-MATS provides a powerful platform for the creation of high fidelity gas turbine engine models, such as the modern turbofan. ${ }^{5-7}$ While the majority of concepts in this paper are general, some are modeling choices as required 
by or reflected in T-MATS.

The following sections of this paper detail the modeling process and performance expectations. Specifically, Section II details the architecture of the turbojet model. Section III describes modeling theory, detailing the Brayton cycle and component level modeling methods. Section IV reviews system level model generation and verifies design point accuracy. An example steady-state performance analysis is conducted in Section V and lastly, a summary and discussion of further topics is located in Section VI.

\section{Model Architecture}

This paper will review engine modeling concepts by detailing the creation of a simple turbojet simulation based on the J85 engine. Data for this example was found in literature ${ }^{2,8,9}$ and consists of performance information at take off and a compressor map. The goal of this model is to match the provided data, while also providing plausible performance data throughout the operational envelope.

The architecture of the turbojet is a compressor, burner, turbine, duct, and nozzle in series, with a single shaft connecting the turbine and compressor, as shown in Fig. 1, which displays the station numbers along the bottom. Inputs to the system include fuel flow $(W f)$ and environmental variables altitude $(A l t), M N$, and delta temperature from standard day $(d T)$. The system output is thrust. Air flow, while technically an input and shown for completeness, is typically calculated internal to the engine model.

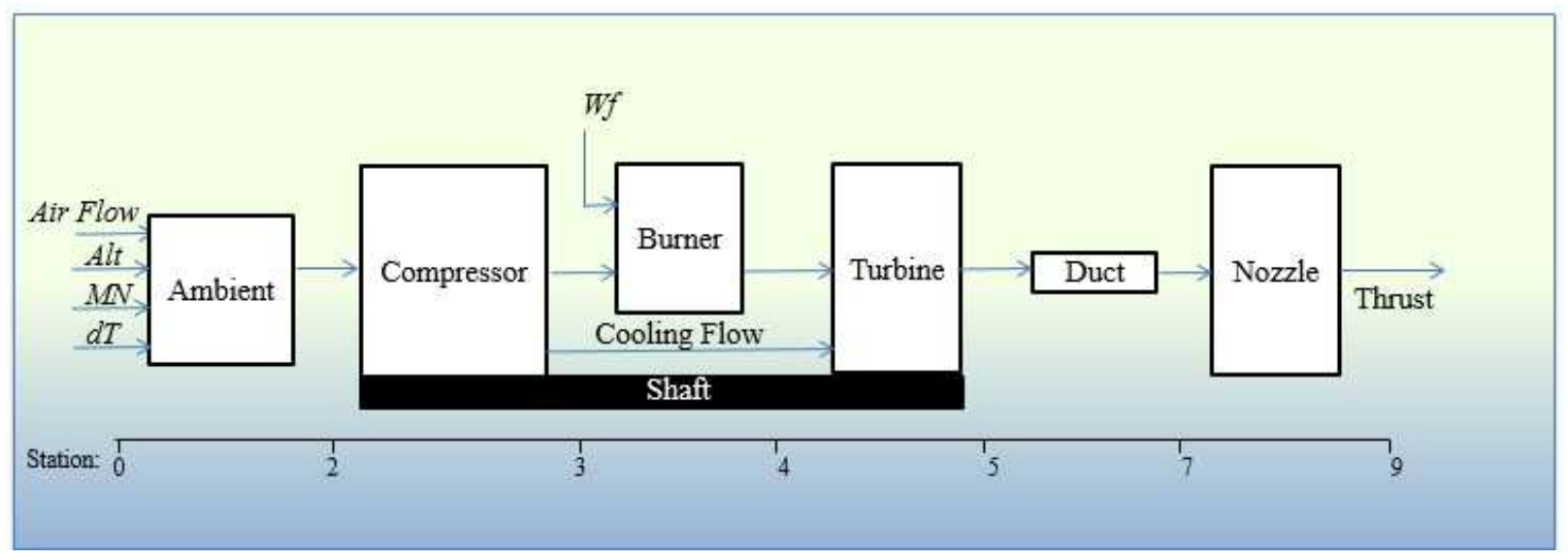

Figure 1. Theoretical J85 system architecture.

Information for the model comes from a combination of published manufacturer and experimental data. Due to various factors (such as possible engine degradation, testing conditions, etc.), discrepancies were found between the data gathered from different sources. For this reason, data for the design were mainly taken from the single largest source ${ }^{2}$ with other variables only used if required and adjusted if needed (as described in detail later in the paper). In general, a take off point is defined when throttle is set to max, determined by a structural limit. This limit is different for various engines, but is typically the maximum pressure at station $3(\mathrm{P} 3)$, shaft speed $(\mathrm{N})$, or temperature at station $5(\mathrm{~T} 5)$ (as a stand in for temperature at station 4 (T4), which can be too hot for the standard measurement techniques used by the control system) that is considered safe for the engine. For the purposes of this paper, $\mathrm{N}$ will be used as the limiting factor for engine operation.

\section{Gas Turbine Modeling Strategy}

Engine modeling has two main components that must be understood, the total system thermodynamics and the component level energy and flow equations. In a thermodynamic gas turbine model, system modeling is based around the Brayton cycle, where a relationship between pressure, temperature, entropy, and enthalpy can be developed. Component modeling is mostly detailed by performance maps that generate key parameters based on the state of the system. Determining the system state requires the use of an external solver that will simultaneously find the operating points on all maps. Information on the use and implementation of these solvers can be found in literature, ${ }^{3,10}$ therefore their operation will not be discussed 
here.

\section{A. Engine System Modeling}

Gas turbine systems can best be described by the thermodynamic cycle known as the Brayton cycle. The ideal Brayton cycle is defined as a thermodynamic cycle that consists of an isentropic and adiabatic compression of a gas, followed by an addition of heat at constant pressure, and an energy extraction that results in gaseous expansion. To understand what the Brayton cycle represents, it is useful to know the Temperature - entropy $(T-s)$ diagram. ${ }^{11}$

The $T$-s diagram shows gas property relationships between temperature $(T)$, entropy $(s)$, and pressure $(P)$. A sample $T-s$ diagram for air has been created with Cantera and is shown in Fig. 2. Looking at the $T-s$ diagram, it can be observed that temperature increases with increasing entropy, and at higher pressures, the temperature to entropy slope is larger. Change in entropy measures the amount of unrecoverable work, while change in temperature directly relates to work that is harnessed. Therefore, the change in temperature to change in entropy ratio for a process should be as high as possible to maximize efficiency. With this in mind, it is no surprise that thermal processes that operate in the higher pressure regions are more efficient.

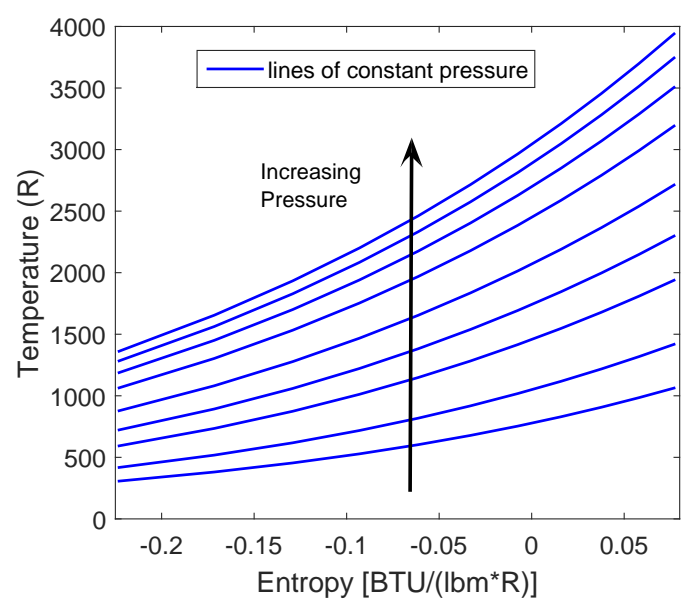

Figure 2. T-s diagram for air, generated with Cantera.

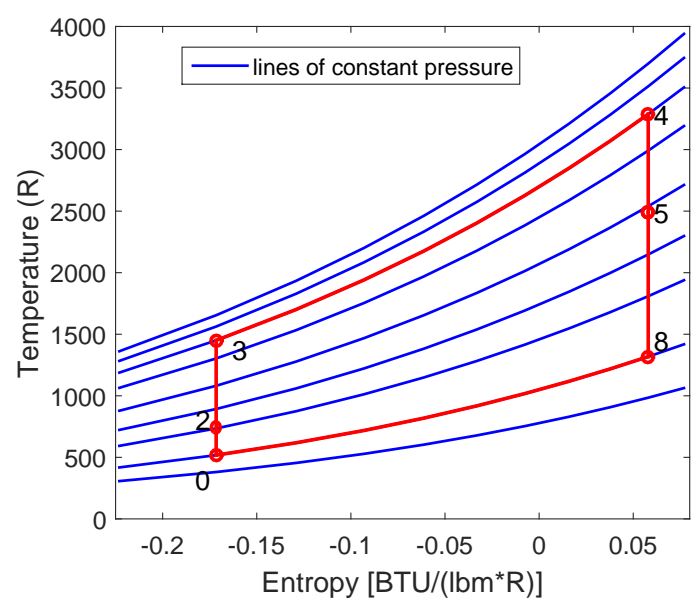

Figure 3. Ideal Brayton Cycle superimposed on $T$-s diagram.

An ideal Brayton cycle superimposed on the $T$-s diagram is shown in Fig. 3. Point 0 represents ambient conditions with pressure and temperature set appropriately. Point 2 represents the conditions at the inlet of the engine. Point 3 represents temperature after compression. It can be noted that the line between Points 0 and 3 results in no rise in entropy, signifying an isentropic expansion where all energy added to the air stream is recoverable. Point 4 shows the temperature and entropy after the addition of heat at constant pressure. Point 5 signifies the temperature after isentropic expansion by the turbine. It should be noted that the amount of work harnessed by the turbine is equivalent to the work added by the compressor, assuming perfect component efficiency. The final point, 8 , shows the cycle returning to ambient pressure, and the length of line 5, 8 is directly related to the amount of work output by the cycle. In the case of an aircraft engine, this work is converted to thrust, however portions may also be used to generate torque or electricity for use on the aircraft. The points described in this section not only mark distinct points in the ideal Brayton cycle, but also coincide with the station numbering scheme used in Fig. 1.

Model fidelity can be increased by accounting for non-ideal, non-isentropic behavior. Earlier in this paper, it was assumed that compression/decompression were isentropic and reversible with no rise in entropy and combustion occurred with no change in pressure. These assumptions reflect an ideal cycle and can introduce significant error into a model. To adjust for these discrepancies, efficiency and pressure loss factors can be added to each component. A non-ideal Brayton cycle with efficiency and pressure loss is shown in Fig. 4. The effects of compression efficiency can be seen as a change in entropy moving from Point 0 to 3 . Similarly, non-ideal turbine efficiency causes a rise in entropy as the cycle moves from Point 4 to 5. Pressure loss in the burner can be seen as Points 3 and 4 are no longer on the same pressure curve. Nozzle efficiency in the 
form of excess pressure and other losses can be noticed as an increase in entropy from Points 5 to 8 . For a complete description of how these factors can be applied, see the sections on component modeling.

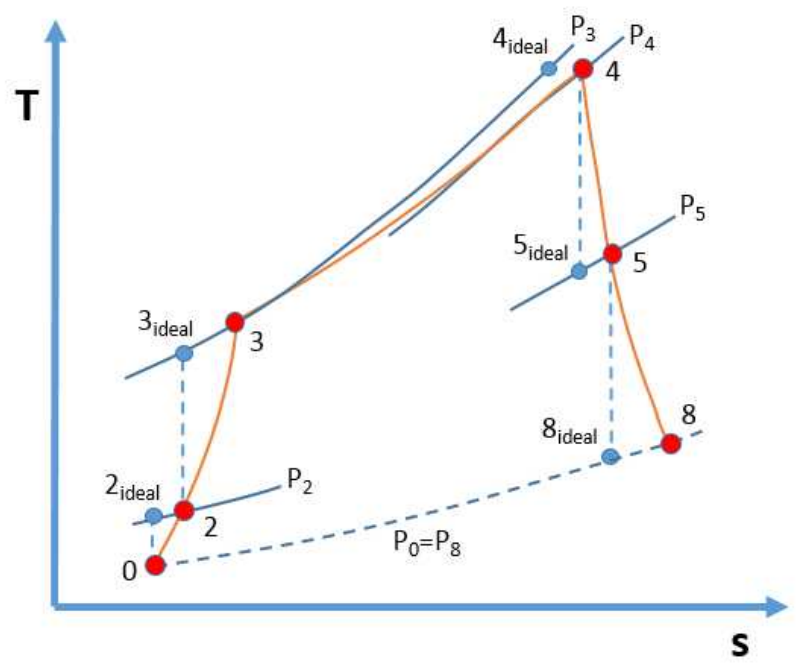

Figure 4. Brayton thermodynamic cycle for a typical single-spool engine. Subscript "ideal" refers to an ideal state, or the end state of an isentropic process.

Values on the $T$-s diagram are typically determined with empirical thermodynamic property tables, high fidelity thermodynamic process codes, or by computation that utilizes thermodynamic relations with a few simplifications. The first major simplification is the assumption that mass flow occurs with an ideal gas where specific heat $\left(C_{p}\right.$ and $\left.C_{v}\right)$ and specific heat ratio $(\gamma)$ are constant. This allows the relationship between change in entropy, pressure, and temperature to be represented as shown in Eq. (1). ${ }^{11}$

$$
s_{f}-s_{i}=C_{p} * \ln \frac{T_{f}}{T_{i}}-R * \ln \frac{P_{f}}{P_{i}}
$$

Assuming isentropic compression or expansion means there is no change in entropy with the process $\left(s_{i}=s_{f}\right)$, and the equation can be simplified, as shown in Eq. (2) and Eq. (3). Recall that $\gamma=\frac{C_{p}}{C_{v}}$ and $R=C_{p}-C_{v}$. This results in a direct relationship between component pressure and temperature ratio with a constant $(\gamma)$.

$$
\begin{gathered}
\ln \frac{T_{f}}{T_{i}}=\frac{R}{C_{p}} \ln \frac{P_{f}}{P_{i}} \\
\frac{T_{f}}{T_{i}}={\frac{P_{f}}{P_{i}}}^{\frac{\gamma-1}{\gamma}}
\end{gathered}
$$

As mentioned above, these equations assume $\gamma$ is constant, however in reality, $\gamma$ is a function of temperature and fluid composition. To determine the accuracy of the isentropic relations with varying temperatures, a control $T$-s diagram was created with the high fidelity thermodynamic process software Cantera, and then compared with calculated values at the various pressure levels. In generating the calculated parameter plots, $T_{f}$ was determined by assuming $\gamma$ was 1.4 , which coincides with standard day conditions, and $P_{i}$ and $T_{i}$ were set equal to ambient conditions. The comparison is shown in Fig. 5. It can be seen that matching is relatively good at low pressure and entropy values, however at higher pressures the assumed $\gamma$ value generates high error values, revealing that the constant $\gamma$ assumption will need to be revised. Updating $\gamma$ by linearly interpolating from 1.39 to 1.31 as a function of $T_{i}$ improves the errors to less than $1 \%$ at all pressures and entropies, as shown in Fig. 6.

Up until this point, all $T$-s property tables have been generated with a single fluid composition (air: $75.47 \%$ nitrogen(N2), $23.2 \%$ oxygen (O2), and $1.28 \%$ argon (AR)), however, in actual gas turbine operation, mass flow after the burner component contains combustion products or exhaust. To determine how the value of $\gamma$ would be affected by the change in chemical composition, an air/exhaust mixture $T$ - $s$ diagram was 


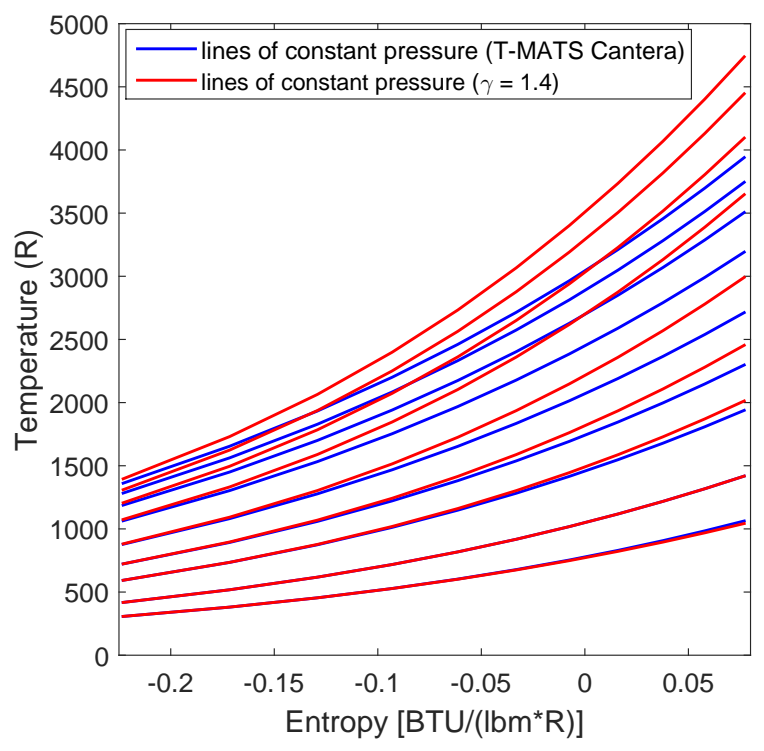

Figure 5. $T$ - $s$ diagram for air comparison, Cantera vs. isentropic relations $(\gamma=1.4)$.

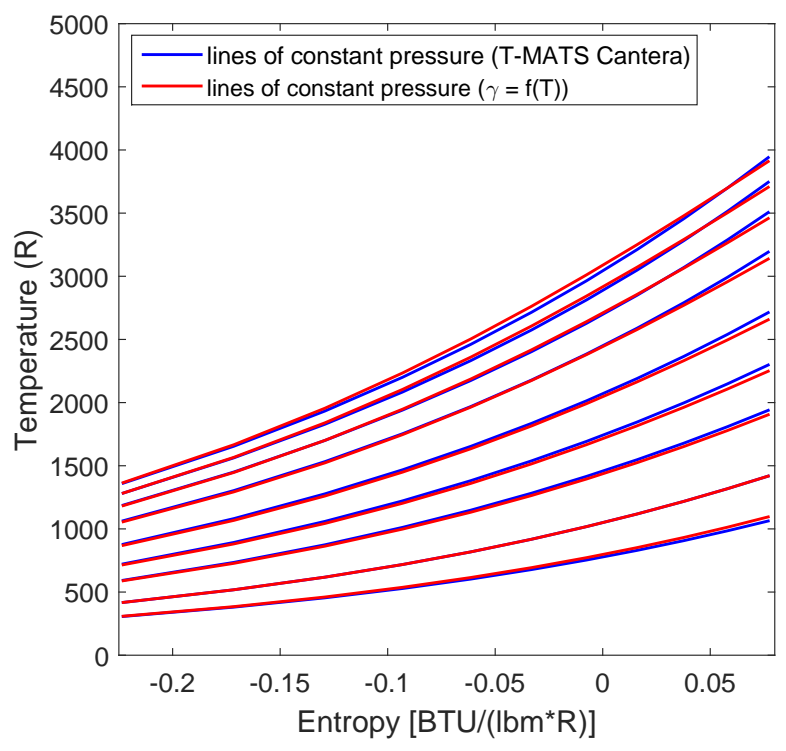

Figure 6. T-s diagram for air comparison, Cantera vs. isentropic relations $(\gamma=1.39$ to 1.31 as function of initial temperature).

generated using Cantera to determine the thermodynamic properties after combustion. For this analysis, combustion was simulated using an arbitrarily selected 0.03 fuel to air ratio (FAR) with fuel properties assumed to be similar to a mixture of $92.2 \%$ methylene $(\mathrm{CH} 2)$ and $7.8 \%$ methylidyne $(\mathrm{CH})$. A comparison of air only and post-combustion $T-s$ diagrams, shown in Fig. 7, reveals fairly small differences at low pressure and entropy values, however at large values there is a significant shift lower in temperature. To account for this shift, the $\gamma$ values must be updated when modeling sections of the gas turbine after the burner, such as the turbine. Comparisons shown in Figs. 5 to 7 illustrate that care should be taken when selecting $\gamma$ for use with the isentropic relation equations. When modeling, adjustments in $\gamma$ required for accurately determining temperature for different gas types and temperature ranges may be gathered into a table format then looked up as needed.

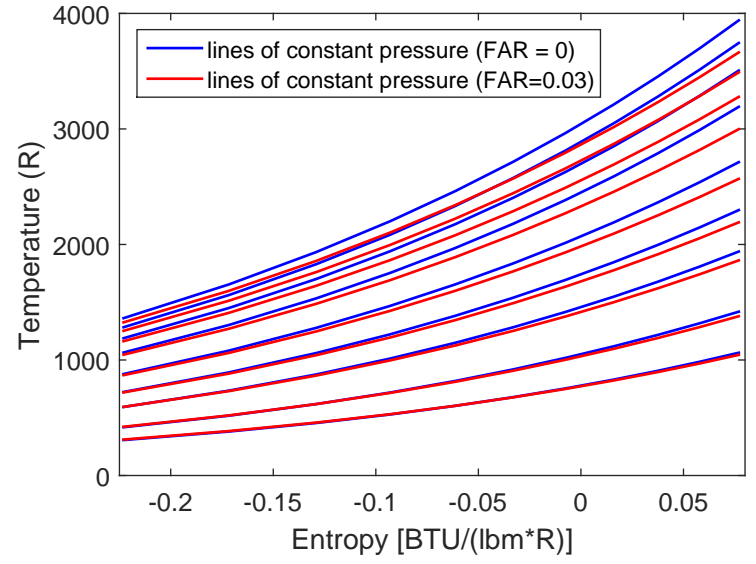

Figure 7. $T$ - $s$ diagram comparison for air and a mixture of air and exhaust $(\mathrm{FAR}=0.03)$.
An alternative to using the $\gamma$ dependent isentropic relation equations is to simply use $T$-s diagram table lookups that offer a direct relationship between pressure, temperature, and entropy. These types of property tables, found in texts or in thermodynamic simulation packages such as T-MATS, ${ }^{3}$ can be generated from physical testing or high fidelity thermodynamic simulation packages, such as Cantera, and can offer very accurate results. Further modeling discussions will make use of these types of gas property tables for calculations, referring to them by entropy and pressure to temperature (sp2t) or pressure and temperature to entropy (pt2s). A similar rational to that used for entropy tables can be used to suggest replacing enthalpy calculations with enthalpy lookup tables. In further explanations of modeling, enthalpy to temperature (h2t) and temperature to enthalpy (t2h) lookup tables will be used in place of thermodynamic equations. 


\section{B. Ambient and Inlet Modeling}

The transition of airflow (mass flow) from ambient or air outside the system to the front of the engine occurs between stations 0 and 2. The key difference between these two stations is that at ambient conditions air is static, and after the inlet it is moving relative to the engine. For subsonic modeling purposes, the changes in mass flow properties are mainly caused by a slowing of the air across the length of the inlet. Generally mass flow, altitude, $M N$, and $d T$ will be known values and can be directly correlated to ambient, static input, pressure, and temperature. Total values can be determined following the process shown in Eq. (4).

$$
\begin{aligned}
& \text { 1) } s_{\text {out }}=s_{\text {in }} \\
& \text { 2) } h s=t 2 h\left(T s_{\text {in }}\right) \\
& \text { 3) } P t_{g}=\text { GuessValue } \\
& \text { 4) } T t_{g}=\operatorname{sp} 2 t\left(s_{\text {out }}, P t_{g}\right) \\
& \text { 5) } h t_{g}=t 2 h\left(T t_{g}\right) \\
& \text { 6) } V_{g}=J C * g * \sqrt{2\left(h t_{g}-h s\right)} \\
& \text { 7) } M N_{g}=\frac{V_{g}}{V s} \\
& \text { 8) } P t_{\text {out Ideal }}, T t_{\text {out }}, V_{\text {out }}=\text { iterate on } P t_{g} \text { until } M N_{g} \text { equals } M N_{\text {in }} \\
& \text { 9) } P t_{\text {out }}=(1-d P) * P t_{\text {out Ideal }} \\
& \text { 10) } F_{d}=\frac{W_{\text {in }} * \text { Vout }}{g}
\end{aligned}
$$

In Eq. (4) dP is used to account for pressure loss across the inlet (typically less than 0.1), and the subscript $g$ indicates a guess value.

The equation statement "iterate on $P t_{g}$ until $M N_{g}$ equals $M N_{i n}$ " is used to specify terms that will need to be solved for iteratively, in this case updating $P t_{g}$ until $M N_{g}=M N_{i n}$. Generally, it has been the experience of the authors that the secant algorithm, ${ }^{2}$ and shown for convenience in Eq. (5) (where $\mathrm{x}$ is the value being solved for and $\mathrm{E}$ is the error between a known value and a determiner $(f(x)))$, is sufficient for solving these types of problems.

$$
x_{n+1}=x_{n}-E_{n} \frac{x_{n}-x_{n-1}}{E_{n}-E_{n-1}}
$$

In the case above, the $x$ term would be $P t_{g}$ and $E$ is $M N_{g}-M N_{i n}$. Once the $\mathrm{E}$ term is driven to zero, $P t_{g}$ and $T t_{g}$ can be used as the actual values.

In supersonic inlets, the geometry of the inlet is used to reduce the velocity of the flow to subsonic values for engine ingestion. While Eq. (4) can provide a rough estimate of supersonic flow ingestion, complications, such as shock formation and engine specific inlet architectures designed to slow the flow, are not taken into account and must be added to the modeling equations. These additional equations are beyond the scope of this paper and will not be discussed further. More information about supersonic inlets can be found in the references. ${ }^{13,14}$

\section{Compressor Modeling}

The compression portion of the cycle occurs between stations 2 and 3 of a gas turbine engine. Modeling compressors can be accomplished by using an empirical performance map. This map develops the relationship between compressor pressure ratio $(P R)$, corrected shaft speed $(N c)$, corrected mass flow $(W c)$, and efficiency, and typically takes into account all compressor variable geometries, such as inlet guide vanes or variable stator vanes. A compressor map can be defined in many different ways, one common way is by defining three sets of 2-D arrays that relate $W c, P R$, and efficiency based on $N c$ and Rline (arbitrarily defined lines on the compressor map that typically lie parallel to the surge line). It should be noticed that this map is defined in terms of corrected speed and mass flow. Correcting speed and mass flow with temperature and/or pressure allows for the creation of a simplified model that is accurate across all operating points. Modeling equations for generating output thermodynamic parameters are shown in Eq. (6). Component pressure ratio and mass flow are taken directly from the map, and output temperature is based on derived 
gas properties and compressor efficiency. The Rline value is selected such that conservation of mass is met (i.e., compressor $W_{\text {in }}=W_{\text {mapDerived }}$ ).

$$
\begin{aligned}
& \text { 1) } s_{\text {out }}=s_{\text {in }}=p t 2 s\left(P t_{\text {in }}, T t_{\text {in }}\right) \\
& \text { 2) } N c_{\text {map }}=N c * s_{-} N c \\
& \text { 3) } W c=\text { maplookup }\left(N c_{\text {map }}, R l i n e\right) * s_{-} W c \\
& \text { 4) } P R=\left(\text { maplookup }\left(N c_{\text {map }}, R l i n e\right)-1\right) * s_{-} P R+1 \\
& \text { 5) } E f f=\text { maplookup }\left(N c_{\text {map }}, R l i n e\right) * s \_E f f \\
& \text { 6) } P t_{\text {out }}=P R * P t_{\text {in }} \\
& \text { 7) } T t_{\text {outIdeal }}=\operatorname{sp} 2 t c\left(s_{\text {out }}, P t_{\text {out }}\right) \\
& \text { 8) } h t_{\text {outIdeal }}=t 2 h\left(T t_{\text {outIdeal }}\right) \\
& \text { 9) } h t_{\text {out }}=h t_{\text {in }}+\frac{h t_{\text {outIdeal }}-h t_{\text {in }}}{E f f} \\
& \text { 10) } T t_{\text {out }}=h 2 t\left(h t_{\text {out }}\right)
\end{aligned}
$$

In Eq. (6) $s_{-} N c, s_{-} W c, s_{-} P R$, and $s_{-} E f f$ are map scalars used for adjusting the compressor map values.

In the modern gas turbine engine, it is common to harvest air from the compressor to be used for cooling or for uses outside the engine. Characteristics for this air, known as bleed air, can be determined as shown in Eq. (7). Fractional bleed constants $\left(C_{h t}\right.$ and $\left.C_{P t}\right)$ act as a linear approximation of inner-stage compressor bleed flow and are set to 1 when bleed is taken from the compressor exit. The fractional flow constant $\left(C_{W}\right)$ is determined by a design point bleed requirement.

$$
\begin{aligned}
& \text { 1) } W_{\text {bleed }}=W_{\text {in }} * C_{W} \\
& \text { 2) } h t_{\text {bleed }}=h t_{\text {in }}+C_{h t} *\left(h t_{\text {out }}-h t_{\text {in }}\right) \\
& \text { 3) } P t_{\text {bleed }}=P t_{\text {in }}+C_{P t} *\left(P t_{\text {out }}-P t_{\text {in }}\right) \\
& \text { 4) } T t_{\text {bleed }}=h 2 t\left(h t_{\text {bleed }}\right)
\end{aligned}
$$

Shaft torque required to generate the specified compressor pressure ratio can be determined based on the total power calculation with any early stage bleed power debit removed, as shown in Eq. (8). In the case where bleed is taken from the exit of the compressor, total compression is required for all of the mass flow entering the compressor, therefore, $h t_{\text {bleed }}=h t_{\text {out }}$, and the debit $P w r_{\text {bleed }}=0$. It should be noted that compressor power values will be negative to denote a usage of power.

$$
\begin{aligned}
& \text { 1) } P w r_{\text {ideal }}=W_{\text {in }} *\left(h t_{\text {in }}-h t_{\text {out }}\right) * B T U p \operatorname{Sec} 2 H P \\
& \text { 2) } P w r_{\text {bleed }}=W_{\text {bleed }} *\left(h t_{\text {bleed }}-h t_{\text {out }}\right) * B T U p \operatorname{Sec} 2 H P \\
& \text { 3) } P w r_{\text {out }}=P w r_{\text {ideal }}-P w r_{\text {bleed }} \\
& \text { 4) } \text { Torque }=\frac{P w r}{N} * H P p R P M 2 F T_{L} B F
\end{aligned}
$$

In Eq. (8) BTUpSec2HP and HPpRPM2FT LBF are unit conversion constants equal to 1.41 and 5252 respectively.

Please note that operational effects from variable geometries, such as variable stator vanes, are typically considered to operate ideally and are taken into account in the compressor map. For more information on compressor variable geometries and their effects see Ref. 1.

\section{Burner Modeling}

Fuel introduction and burning occurs from station 3 to 4 . In modeling the burner, a total burn assumption can be made where all fuel entering the chamber is burned in its entirety. The energy in the fuel is modeled with a flow amount and a lower heating value (LHV). For jet fuel the LHV can be assumed to have a value close to 18,400 BTU/lbm. Energy equations used for the burner model can be seen in Eq. (9). It should be noted that although this is considered a constant pressure process, there may be a slight pressure loss, $d P$, (1\% to $5 \%$ ) associated with flow moving through the complicated orifices of the burner. 


$$
\begin{aligned}
& \text { 1) } W_{\text {out }}=W_{\text {in }}+W f \\
& \text { 2) } h t_{\text {out }}=\frac{W_{\text {in }} * h t_{\text {in }}+W f * L H V * E f f}{W_{\text {out }}} \\
& \text { 3) } T t_{\text {out }}=h 2 t\left(h t_{\text {out }}\right) \\
& \text { 4) } P t_{\text {out }}=(1-d P) * P t_{\text {in }}
\end{aligned}
$$

In Eq. (9) $E f f$ is burner efficiency.

\section{E. Turbine Modeling}

The turbine component of the cycle is located between stations 4 and 5 of a gas turbine engine. Similar to the compressor, the turbine is modeled using a performance map. This map correlates the relationships between $P R, W c, N c$, and efficiency, and will generally take the form of two 2-D tables that determine $W c$ and efficiency based on $P R$ and $N c$. Conservation of mass is met by adjusting $P R_{i n}$ until $W_{i n}=W_{\text {mapDerived }}$. Modeling equations that assume no cooling flow are shown in Eq. (10).

$$
\begin{aligned}
& \text { 1) } s_{\text {out }}=s_{\text {in }}=p t 2 s\left(P t_{\text {in }}, T t_{\text {in }}\right) \\
& \text { 2) } N c_{\text {map }}=N c * s_{-} N c \\
& \text { 3) } P R_{\text {map }}=\frac{P R-1}{s_{-} P R}+1 \\
& \text { 4) } W c=\text { maplookup }\left(N c_{\text {map }}, P R_{\text {map }}\right) * s_{-} W c \\
& \text { 5) } E f f=\text { maplookup }\left(N c_{\text {map }}, P R_{\text {map }}\right) * s_{-} E f f \\
& \text { 6) } P t_{\text {out }}=P R * P t_{\text {in }} \\
& \text { 7) } T t_{\text {outIdeal }}=\operatorname{sp} 2 t c\left(s_{\text {out }}, P t_{\text {out }}\right) \\
& \text { 8) } h t_{\text {out Ideal }}=t 2 h\left(T t_{\text {out Ideal }}\right) \\
& \text { 9) } h t_{\text {out }}=h t_{\text {in }}+E f f *\left(h t_{\text {in }}-h t_{\text {outIdeal }}\right) \\
& \text { 10) } T t_{\text {out }}=h 2 t\left(h t_{\text {out }}\right)
\end{aligned}
$$

In Eq. (10) $s_{-} N c, s_{-} W c, s_{-} P R$, and $s_{-} E f f$ are map scalars used for adjusting the turbine map values.

Turbine cooling flow may be inserted before or after the core flow has moved through the turbine, by directly adding the $W_{\text {bleed }}$ from the compressor as $W_{\text {cool }}$. The $h t$ is then updated based on the mass flow weighted averages, and $P t$ is assumed to be unchanged from the core or input flow value, as shown in Eq. (11). While this method greatly simplifies the mixing process, the authors have found it to be sufficient in cases with low relative cooling flow.

$$
\begin{aligned}
& \text { 1) } W_{\text {out }}=W_{\text {in }}+W_{\text {cool }} \\
& \text { 2) } h t_{\text {out }}=\frac{h t_{\text {in }} * W_{\text {in }}+h t_{\text {cool }} * W_{\text {cool }}}{W_{\text {in }}+W_{\text {cool }}} \\
& \text { 3) } T t_{\text {out }}=h 2 t\left(h_{\text {out }}\right) \\
& \text { 4) } P t_{\text {out }}=P t_{\text {in }}
\end{aligned}
$$

Power values for the turbine are calculated exactly the same as for the compressor (Eq. (8)), however, in this case the torque and power values will be positive to denote a generation of power.

\section{F. Duct Modeling}

In many cases, high loss sections of the engine, such as long ducts, may be represented by a simple pressure drop to account for frictional losses. In the turbojet example, one such section is the duct leading from the turbine to the nozzle. Formulation of a duct section can be seen in Eq. (12).

$$
P t_{\text {out }}=(1-d P) * P_{\text {in }}
$$

In Eq. (12) $d P$ is the pressure loss term. Pressure loss across the section can range fairly widely, but is typically less than 0.02 . 


\section{G. Nozzle Modeling}

The aft section of an aircraft engine, stations 7 to 9 , is typically made up of a nozzle. This nozzle translates excess pressure into thrust with the use of a convergent duct, followed in some cases by a divergent duct. Commercial engines almost exclusively use a convergent nozzle. There are many ways that the performance of a nozzle can be modeled, most methods calculate ideal pressure $\left(P_{t h}=P_{a m b}\right)$ or velocity $\left(V_{\text {out }}=V_{M N 1}\right)$ values at the throat or exit, then apply coefficients to account for non-ideal behavior. This paper will review the use of the coefficient of thrust $\left(C_{f g}\right)$ that utilizes the ideal pressure method to determine thrust. This coefficient can be defined as a function of nozzle pressure ratio, and takes into account nozzle losses due to friction, angularity, expansion, leakage, and cooling air throttling loss. ${ }^{13}$ The full nozzle thrust model can be seen in Eq. (13).

1) $s_{\text {out }}=s_{\text {in }}$

2) $T s_{\text {outIdeal }}=\operatorname{sp} 2 t\left(s_{\text {out }}, P_{\text {amb }}\right)$

3) $h s_{\text {outIdeal }}=t 2 h\left(T s_{\text {outIdeal }}\right)$

4) $V_{\text {outIdeal }}=J C * g * \sqrt{2\left(h t_{\text {in }}-h s_{\text {outIdeal }}\right)}$

5) $C_{f g}=\frac{F g_{\text {actual }}}{F g_{\text {Ideal }}}$

6) $F_{g}=\frac{W_{\text {in }} * V_{\text {outIdeal }}}{g} * C_{f g}$

7) $F_{n}=F_{g}-F_{d}$

In addition to calculating thrust, the nozzle component also calculates a total mass flow that is used to determine the flow for the entire system. ${ }^{3}$ This nozzle flow is calculated utilizing ideal throat calculations Eq. (13) and the equations shown in Eq. (14).

$$
\begin{aligned}
& \text { 1) } \rho_{\text {outIdeal }}=\frac{P_{a m b} * P S I 2 P S F}{R * T s_{\text {outIdeal }} * J C} \\
& \text { 1) } C_{D}=\frac{W_{\text {actual }}}{W_{\text {Ideal }}} \\
& \text { 1) } W_{\text {outCalc }}=C_{D} * \frac{\rho_{\text {outIdeal }} * \min \left(V_{\text {outIdeal }}, V_{M N 1}\right) * A_{t h}}{P S I 2 P S F}
\end{aligned}
$$

In Eq. (14) PSI2PSF is a unit conversion factor equal to $144, C_{D}$ is the coefficient of drag that accounts for non-ideal flow behavior, ${ }^{13}$ and $V_{M N 1}$ is flow velocity at Mach number equal to 1 , which can be calculated using Eq. (4). In a convergent nozzle, flow will accelerate as exit area is reduced but only until the speed of the flow reaches Mach 1, at which point it is considered choked and flow speed remains constant. For the thrust calculation, this effect is taken into account by the thrust coefficient, but for the flow calculation it needs to be accounted for directly by comparing the choked velocity $\left(V_{M N 1}\right)$ with the idealized pressure calculated velocity ( $V_{\text {outIdeal }}$ generated by assuming $\left.P_{a m b}=P_{t h}\right)$. When $V_{M N 1}>V_{\text {outIdeal }}$ the nozzle is considered unchoked, otherwise it is choked.

A nozzle designed to accelerate exit air past $M N=1$ is typically referred to as a convergent-divergent nozzle, where the convergent section speeds the flow to $M N=1$ then the divergent section increases the speed further into the supersonic regime. This type of nozzle is utilized on supersonic aircraft applications and will not be discussed further in this paper. However, additional information on modeling convergentdivergent nozzles can be found in Ref. 12 and 13. 


\section{H. Shaft Modeling}

In a gas turbine model, the shaft component connects the turbomachinery components generating power with those consuming power. In a single spool engine, all components are connected via a single shaft, and in a dual spool engine, the components are divided between two shafts. When developing a steady-state model, no shaft dynamics need to be taken into account. The torque can simply be balanced. However, for completeness, the procedure for adding inertia terms is laid out in Eq. (15).

$$
\begin{aligned}
\frac{\partial N}{\partial t} & =\frac{\sum T r q_{\text {compressor }}+\sum T r q_{\text {turbine }}+\sum T r q_{\text {other }}}{2 * \pi * I} \\
N & =\int \frac{\partial N}{\partial t}
\end{aligned}
$$

In Eq. (15) $\operatorname{Trq}$ is component torque, $I$ is shaft inertia, and $\frac{\partial N}{\partial t}$ is equal to zero during steady-state operation.

\section{Model Generation}

Once the system architecture was decided upon (shown in Section II), the performance model of the J85 engine was created using T-MATS. A diagram of the steady-state turbojet system is shown in Fig. 8. The use of a thermodynamic modeling package, such as T-MATS, simplifies model generation by packaging component level calculations into easy to manage blocks. This type of high level modeling reduces the immediate need for understanding the low level equations required for engine model creation allowing for a quick development process.

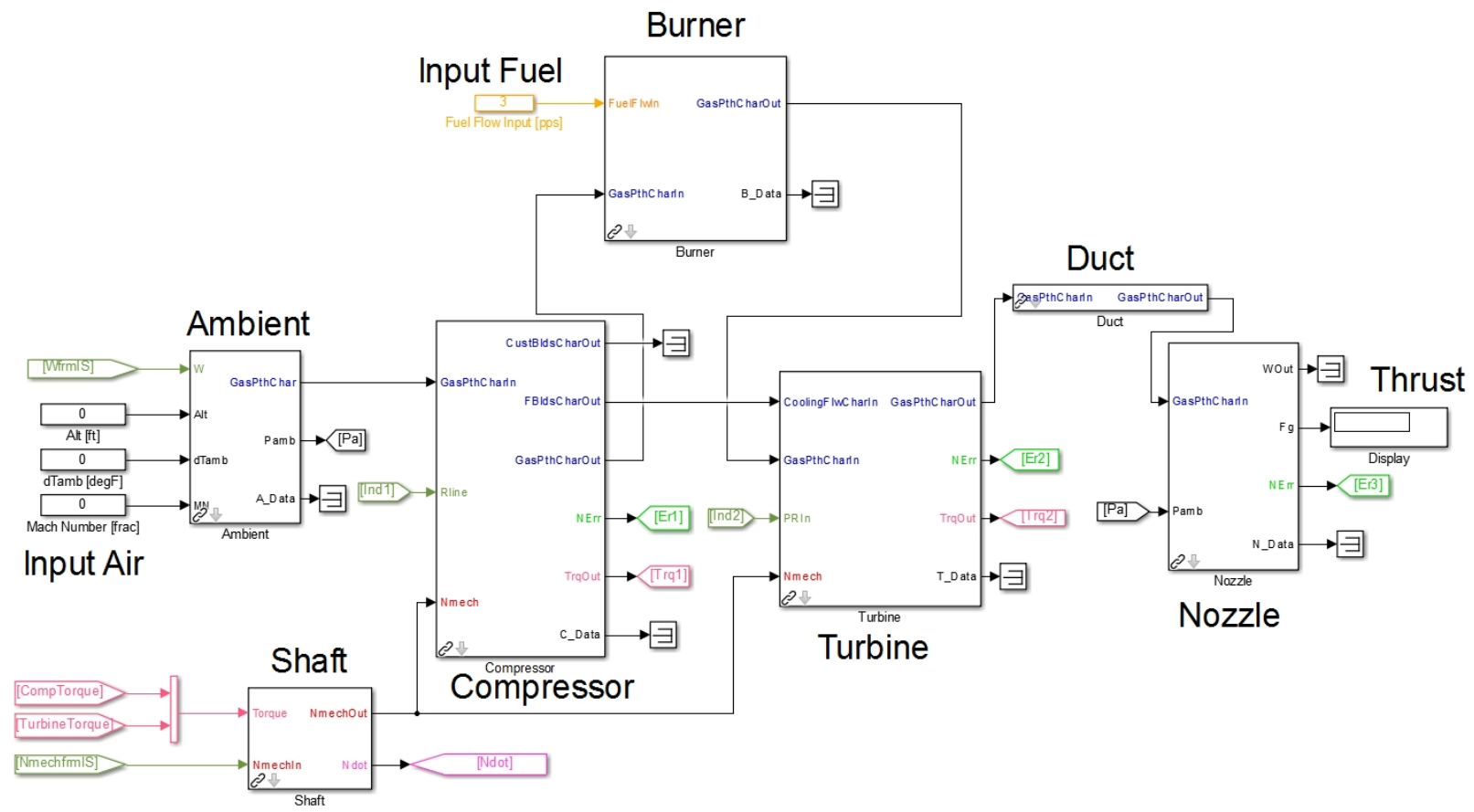

Figure 8. T-MATS turbojet model.

Data taken from literature to be used for model generation are located in Table 1, and describes a take off condition or design point. Design point or on-design data refers to a mission critical operating point that the engine was specifically designed to meet, typically takeoff and cruise. Conversely, off-design data refers to performance data generated outside of the specified design points. For this example, the engine was assumed to be shaft speed limited, meaning engine operation is limited by a certain shaft speed value while running at maximum power design points, such as take off. This assumption allows the design limit shaft speed to be set to the same value as the design data shaft speed shown in Table 1. 
Table 1. Design data and engine limits. ${ }^{2,8}$

\begin{tabular}{|c|c|}
\hline Conditions & Takeoff \\
\hline altitude, $A l t$ & $0 \mathrm{ft}$ \\
\hline temperature, $T$ & standard day \\
\hline Mach number, $M N$ & 0 \\
\hline Design Data & \\
\hline net thrust, $F_{\text {net }}$ & $2,850 \mathrm{lbf}$ \\
\hline specific fuel consumption, $S F C$ & $0.99(\mathrm{lbm} / \mathrm{hr}) / \mathrm{lbf}$ \\
\hline shaft speed, $N$ & $16,540 \mathrm{rpm}$ \\
\hline mass flow, $W$ & $44 \mathrm{pps}$ \\
\hline compressor pressure ratio, $P R$ & 7 \\
\hline turbine inlet temperature, $T 4$ & $2,100 \mathrm{R}$ \\
\hline Design Limits & \\
\hline$N_{\max }$ & $16,540 \mathrm{rpm}$ \\
\hline
\end{tabular}

To create the design point model, performance parameters such as pressure ratios, efficiencies, areas, etc. for each engine component are required to be defined. This definition process was iterative, using known values and assumptions to calculate the unknown engine parameters, then making adjustments to previous assumptions as needed to meet the design point, detailed in Table 1.

First, the compressor pressure ratio was taken from the design point data, and compressor efficiency was set based on typical compressor performance. For this study, it was also assumed LHV in Eq. (9) was 18,400 $B T U / \mathrm{lbm}$ and there was no cooling flow. Burner efficiency was then adjusted until the temperature gains due to isentropic compression and fuel burn met the design turbine inlet temperature $(2,100 R)$.

Next, turbine and nozzle parameters were defined. Nozzle input temperature was determined by first back calculating nozzle throat velocity from design thrust and total mass flow ( $W_{\text {total }}=W f+W$, where $W f$ was calculated from the $S F C$ equation, Eq. (16)) and assuming $C_{f g}$ was 1, with equations described in Eq. (14). Once a velocity was determined, nozzle input enthalpy and temperature were calculated. Enthalpy at the nozzle exit was determined utilizing burner entropy, FAR, and ambient pressure.

$$
S F C=\frac{W f * 3,600}{F_{n}}
$$

Finally, Turbine pressure ratio was calculated based on the temperature rise due to isentropic expansion across the turbine assuming an $85 \%$ efficiency. Turbine and compressor torques were also calculated and compared. This comparison showed a greater than $5 \%$ difference between the two torques and greater than $1 \%$ error in certain design point performance values.

To remove torque error, values for burner, turbine, and compressor efficiencies, turbine and duct pressure ratios, the coefficient of thrust, fuel flow, and cooling flows were re-tuned manually. At each iteration, parameter calculations and torque balancing was performed using the T-MATS tool, IDesign. This tool automatically adjusted inputs until compressor and turbine shaft torques cancelled out (steady-state modeling requirement), and after iterating other system inputs all other parameters matched the design point, specifically $S F C$, turbine inlet temperature, shaft speed, and thrust. Final modeling values are broken down by component shown in Table A1 of the Appendix. Design matching errors can be viewed in Table 2, and show a very good match for each design parameter, with errors less than $1 \%$ in all cases.

For design point analysis, performance maps are not needed. Pressure ratios, efficiencies, speeds, and flows are all considered known values or are derived for a specified operating condition. Performance maps are required to move the model to off-design points. For this project, a performance map for the J85 was found in literature. ${ }^{8}$ By inspection it was determined the number of values and range of the map would not cover the envelope as required for this paper, so a second generic map was introduced to complete unknown portions. To merge the two maps, map scaling was performed on the generic map to resize it for the design point. Map scaling is the practice of multiplying each map value by a scalar to force that particular value to line up with the design value. ${ }^{15}$ For our example, a generic map has $N c_{\text {map }}$ values ranging from 0.5 to 1.02 , but the engine's design point $N c$ is $16,540 \mathrm{rpm}$. To gain more realistic numbers from the generic 
Table 2. Design data vs. modeled data.

\begin{tabular}{|c|c|c|c|}
\hline Parameter & Design Data & Modeled & Error \\
\hline thrust & $2,850 \mathrm{lbf}$ & $2,861 \mathrm{lbf}$ & $0.3 \%$ \\
\hline specific fuel consumption & 0.99 & 0.9901 & $0.01 \%$ \\
\hline shaft speed & $16,540 \mathrm{rpm}$ & $16,540 \mathrm{rpm}$ & 0 \\
\hline air flow & $44 \mathrm{pps}$ & $44 \mathrm{pps}$ & 0 \\
\hline compressor pressure ratio & 7 & 7 & 0 \\
\hline turbine inlet temperature & $2,100 \mathrm{R}$ & $2,097 \mathrm{R}$ & $0.14 \%$ \\
\hline
\end{tabular}

map $N c_{\text {map }}$ can be multiplied by a scalar to achieve the design point value, in this case $16,540 \mathrm{rpm}$. To perform these types of scalings accurately, the design point location on the map must be known. To gain an understanding of this location, it is suggested that map scaling first be done about the takeoff point to gain engineering insight. This experience will be helpful in obtaining the cruise design point that is typically more cumbersome to determine. In our example case, the takeoff design point was estimated to be on the 1.00 or $100 \%$ speed line just below the crux of the curve as shown in Fig. 9, and each generic map value $\left(N c_{\text {map }}, P R_{\text {map }}, E f f_{\text {map }}\right.$, and $\left.W c_{\text {map }}\right)$ was scaled to the design value. It should be noted that only the portion of $P R$ above 1 is used for this type of scaling (i.e., $P R=\left(P R_{\text {map }}-1\right) *$ scalar +1$)$. A comparison between the actual compressor map and a generic map scaled to the design point is shown in Fig. 9. It can be seen that there is a fairly good matching close to design speed (1.0 constant speed line), however as shaft speed is adjusted away from the design speed, pressure ratios and airflows diverge significantly. This comparison shows the potential issues when attempting to use generic maps for unknown engine components, and demonstrates why it is preferable to use maps designed for a specific engine.

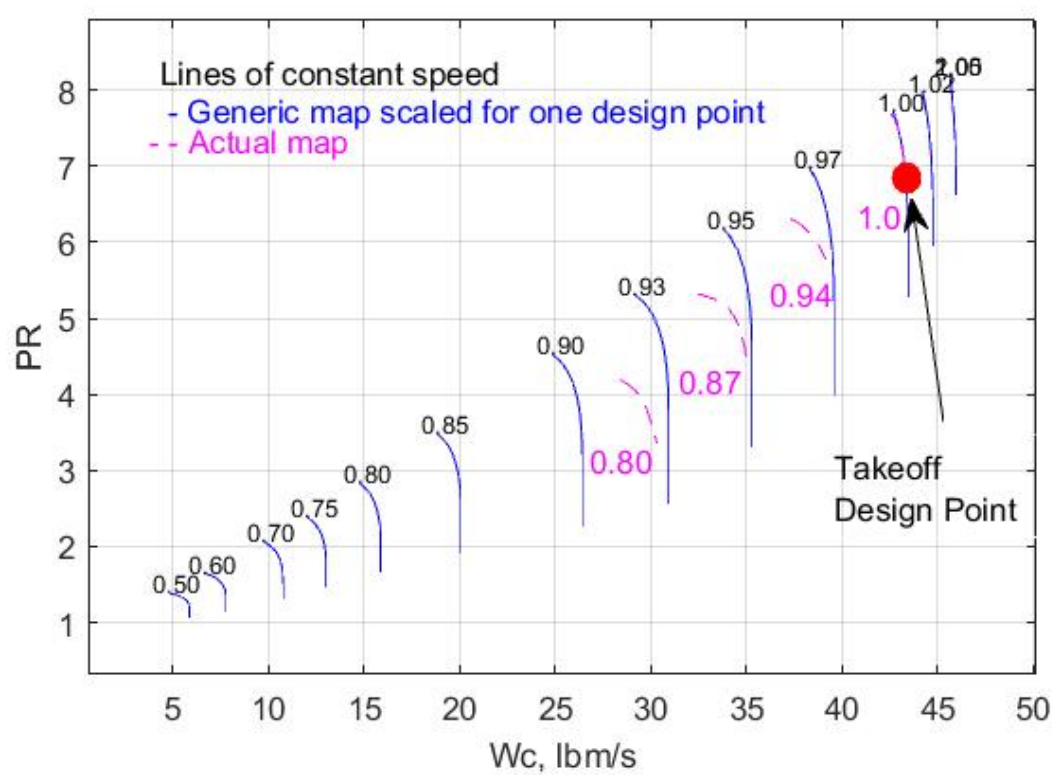

Figure 9. Actual compressor map vs. generic compressor map scaled to design point. ${ }^{3,8}$

To improve compressor map matching, scaling as a function of shaft speed was applied to the generic engine map until the actual map coincided with the generic map, as shown in Fig. 10. In this way, the form of the actual map was utilized while also making use of the expanded generic map. It should be noted that at speeds below $80 \%$, there was no actual map data, therefore this study will only make use of speed points above the $80 \%$ mark. Efficiency values were assumed to be fairly consistent between the two maps with highest efficiencies near the design point and a loss of efficiency as $P R$ and speed decrease. In addition to compressor maps, the model also required a turbine map. In this case a generic map was utilized with a single design point scaling. Unlike the compressor map, actual turbine maps were not found in literature, so 


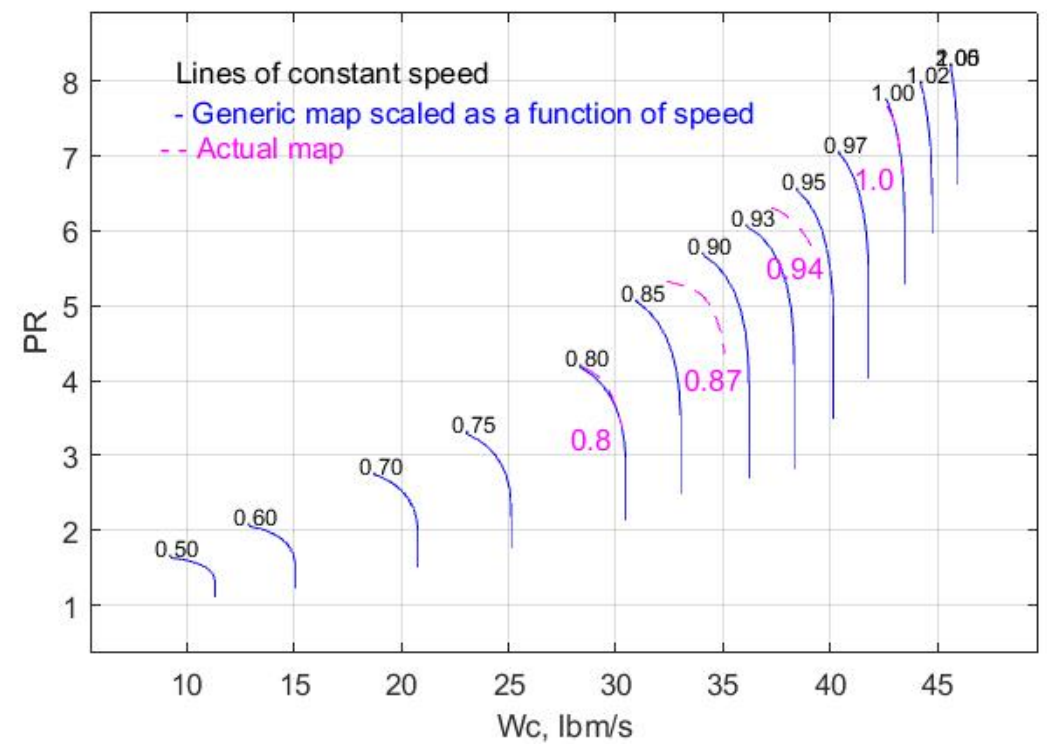

Figure 10. Actual compressor map vs. generic compressor map scaled as function of $N c .^{3,8}$

a direct comparison with an actual map was not possible, and the off-design error will be unknown. These unknowns are deemed acceptable because a turbine typically operates in the choked region, which means shifts in shaft speed will only cause minor changes in corrected mass flow, as shown in Fig. 11. This fluid effect simplifies the relationship between map parameters, resulting in a high degree of similarity between on-design and off-design running. However, when operating in the unchoked region of the turbine map, the constant corrected mass flow assumption cannot be made and an actual engine map may differ from a generic map significantly.

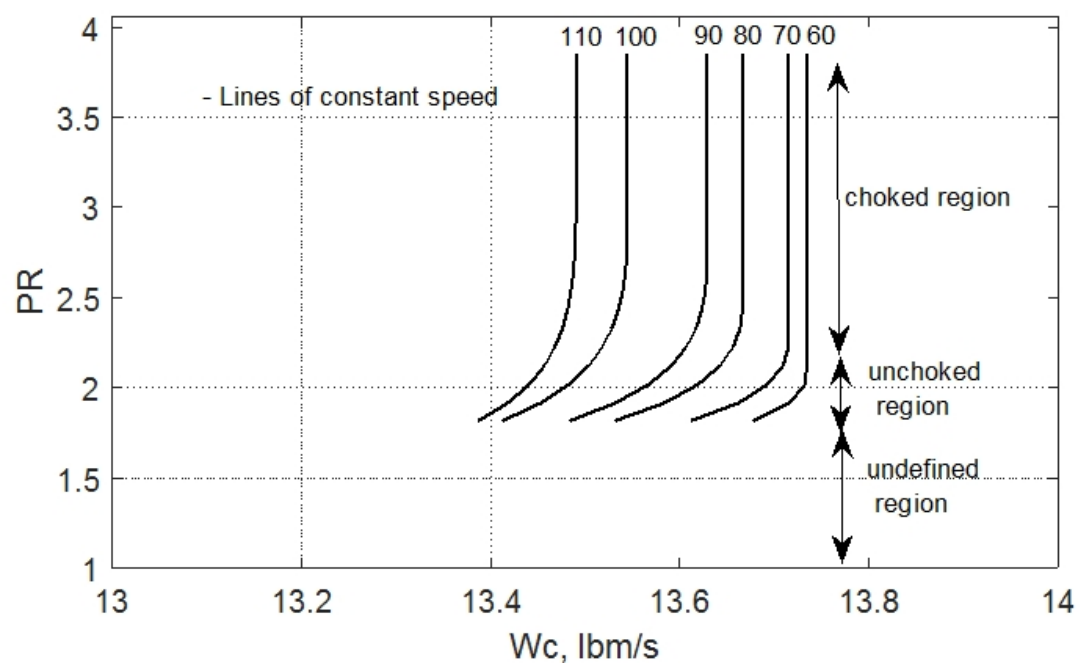

Figure 11. Scaled generic turbine map. ${ }^{3}$

\section{Performance Analysis}

In the previous section, a model of the J85 engine was created using references from literature. A subsequent verification was performed by matching modeled parameters with design point criteria and per- 
formance maps. In this section a performance analysis will be presented using the developed engine model. The purpose of this analysis is to predict engine performance characteristics across the operating envelope. This analysis will delve into two causes of performance variation: location within the envelope, and power level. It should be noted that these two categories are coupled, however trends between them can be extrapolated to the different cases. For this paper, $S F C$ and thrust will be analyzed at max power settings for each envelope condition. Operation at the cruise and sea level static (SLS) conditions will then be used to investigate operational lines and the relationships between $E P R$ or $N c$ and thrust.

The operational envelope was defined as the altitude and Mach numbers at which the engine is expected to operate. With a max altitude of 35,000 ft and speed of $500 \mathrm{mph}$, the Viper Jet MKII homebuilt airplane is known to use the J85 as it's power plant ${ }^{16}$ and was used to set the envelope requirement for this paper. Steady-state points that were considered in this analysis appear in Fig. 12 and are enclosed in the flight envelope. Portions of the flight envelope rarely used, such as low $M N$ at high altitude and high $M N$ at low altitude, were removed for convenience purposes.

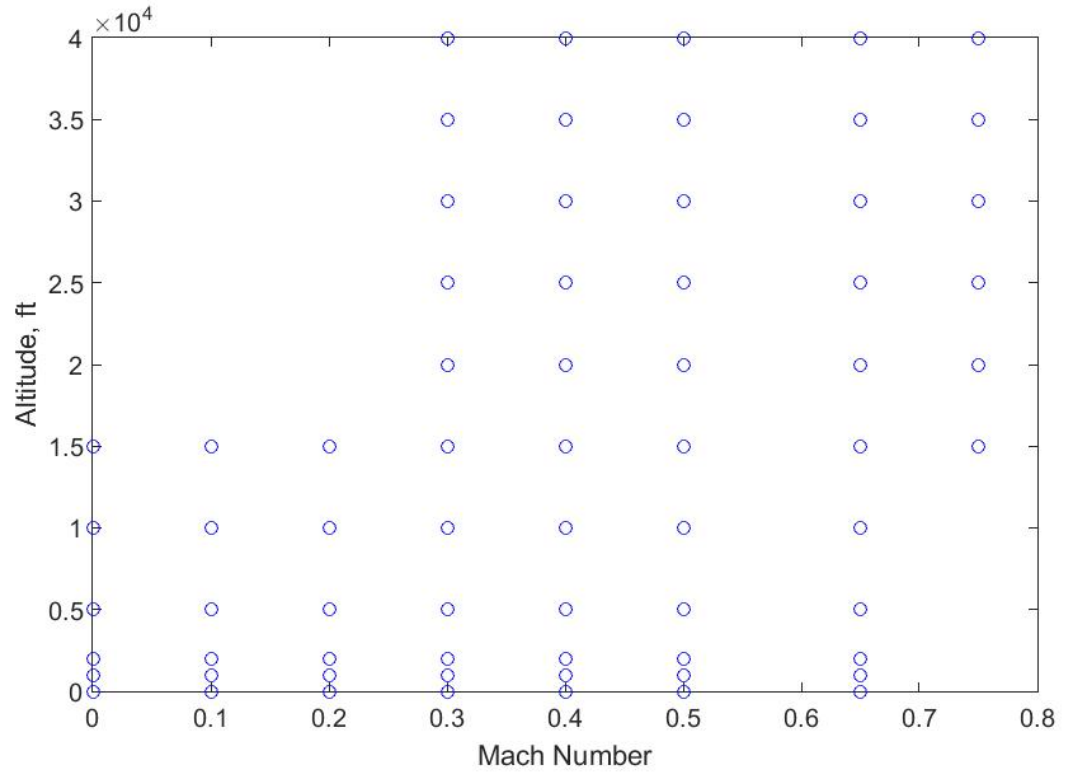

Figure 12. Flight envelope.

The predicted $S F C$ given in Fig. 13 shows significant increase in fuel consumption as Mach number is increased or as altitude is decreased. This $S F C$ rise can be attributed to the higher drag values at larger Mach number and the rise in air density with lower altitudes, where more power is needed to push dense air through the engine. Predicted max net thrust for the simulated J85 engine is given in Fig. 14. It can be seen that as altitude is increased, thrust decreases. These lower thrust values can largely be attributed to the decrease in air density as elevation is increased. A lower density reduces the mass flow achieved for a given shaft speed, leading to smaller thrust values as defined by Eq. (13) and (14). Additionally, as Mach number is increased from 0 , net thrust reduces due to ram drag effects. As Mach number is increased further, thrust begins to increase as the pressure gains in the engine contribute to thrust gains proportionally larger than the increase in drag. Generally these results lead to greater thrust at low altitude/higher speeds, and to better fuel economy at high altitudes/lower speeds. This $S F C$ advantage has limits however, as altitude becomes very high, the air density may become so low that a typical airplane may not generate enough lift to maintain altitude, and the engine could blow out or stall. These altitude limits are determined during the aircraft design phase. For a typical commercial engine, the max altitude will be between 35,000 ft to 40,000 $\mathrm{ft}$ and will coincide with the cruise altitude.

Engine operation at partial power levels can be characterized by looking at the compressor operational lines or op-lines. Op-lines effectively define the pumping characteristics of the engine, ${ }^{2}$ and can be used to visualize engine operation. For this analysis, the engine was de-throttled at two distinct envelope points, SLS and cruise (0.8 MN and 35,000 ft altitude). Throttling for each case consists of small steps between full 


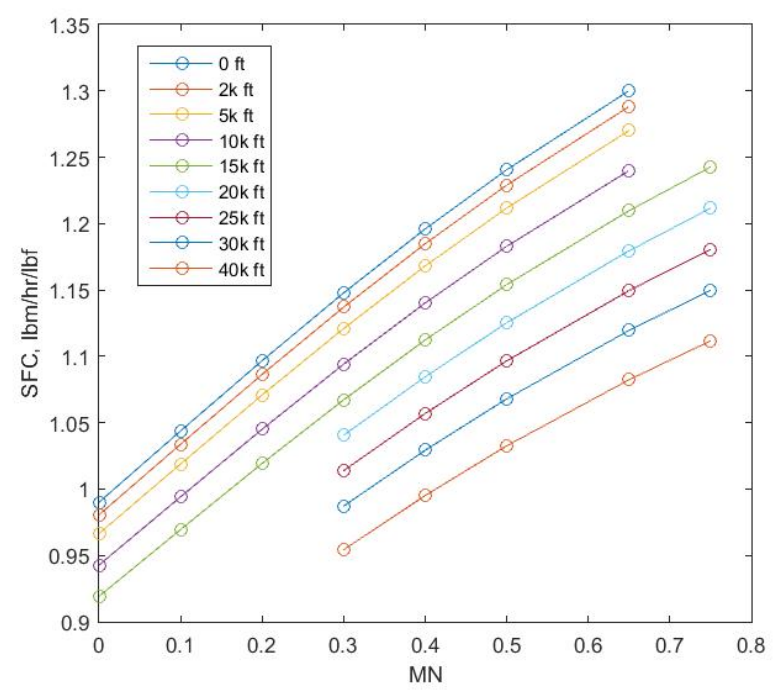

Figure 13. Model $S F C$ vs. $M N$ and altitude.

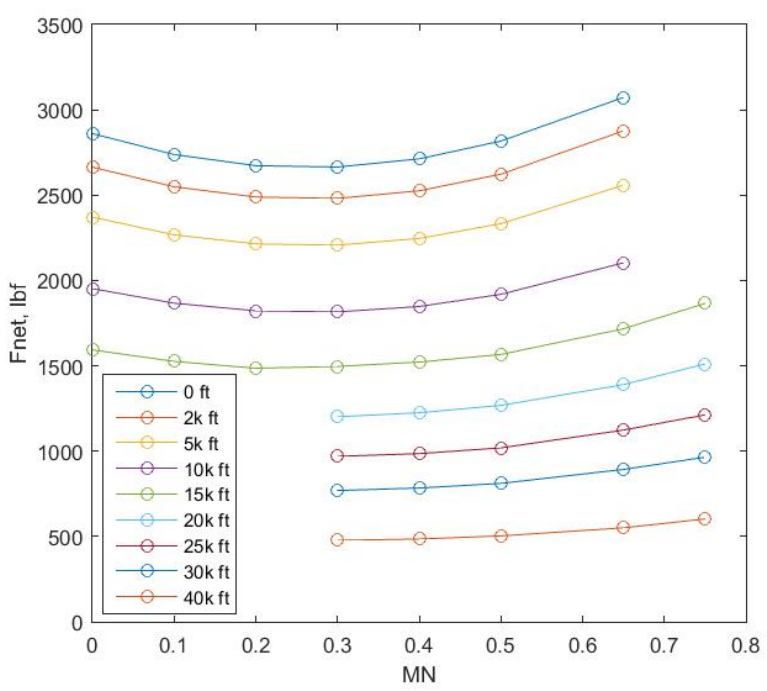

Figure 14. Model $F_{n e t}$ vs. $M N$ and altitude.

power and idle, $100 \%$ and $80 \% N c$ respectively, allowing the engine to reach steady-state between throttle changes. The compressor op-lines, shown in Fig. 15, relate the results from the two throttling shifts. It can be seen that the op-lines are coincident at high power levels, and at lower power levels they begin to diverge. In general, op-lines are consistent across the envelope when engine geometry or state remains consistent. For example, increasing nozzle area, increases compressor mass flow and $P R$, and pushing the op-line down and to the right as the pumping characteristic of the engine is shifted. Similar to area changes, the choked state

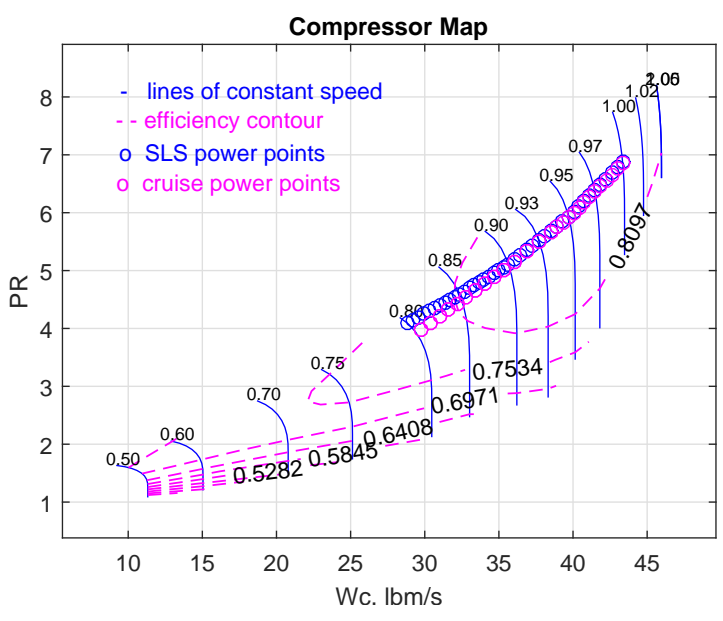

Figure 15. Compressor op-lines at SLS and cruise.

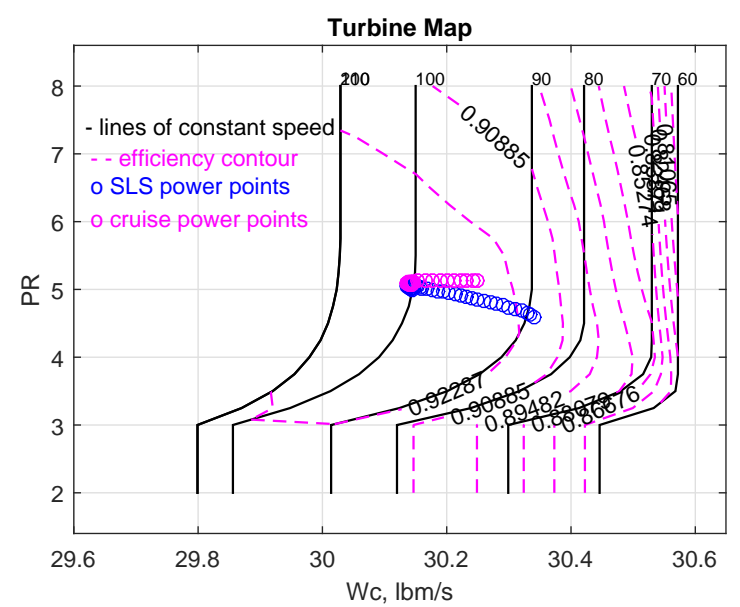

Figure 16. Turbine operational points at SLS and cruise.

of the nozzle can also affect the op-line. In an unchoked nozzle, the nozzle exit pressure becomes a function of ambient pressure rather than engine operating pressure shifting the op-line. In Fig. 15, diverging compressor op-lines can be attributed to the unchoking of the nozzle at the SLS lower power level condition. Similar to the compressor op-lines, turbine operational points for the various power levels are given on Fig. 16. Looking at the figure, it can be seen that the $P R$ remains constant at cruise conditions, and reduces slightly as power level is reduced at SLS conditions.

In addition to understanding the above relationships, it is also useful to understand how key measurable engine parameters are related to the engine output thrust. For engine control purposes, the relationships between thrust and engine pressure ratio $(E P R)$ or $N c$ are particularly important. $E P R$ and $N c$ are plotted for a variety of power levels at the two operational points discussed above, as shown in Figs. 17 
and 18. Looking at the EPR and $N c$ plots, it can be seen that environmental conditions shift thrust levels significantly. This is expected from the plots seen before, however it can also be seen that $E P R$ and thrust have a linear relationship, while $N c$ has a fairly simple relationship with thrust. This is important for controls because thrust cannot be measured, so a measurable signal must be used as a stand in when setting up the feedback for the control system. Generally, this signal is either EPR or $N c$ (for a single spool engine) because of their simple relationships with thrust.

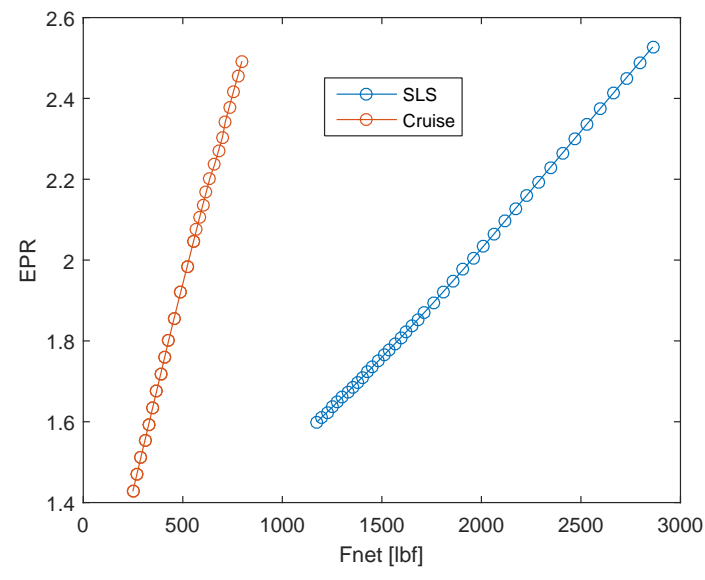

Figure 17. EPR vs. $F_{n}$, idle to max power.

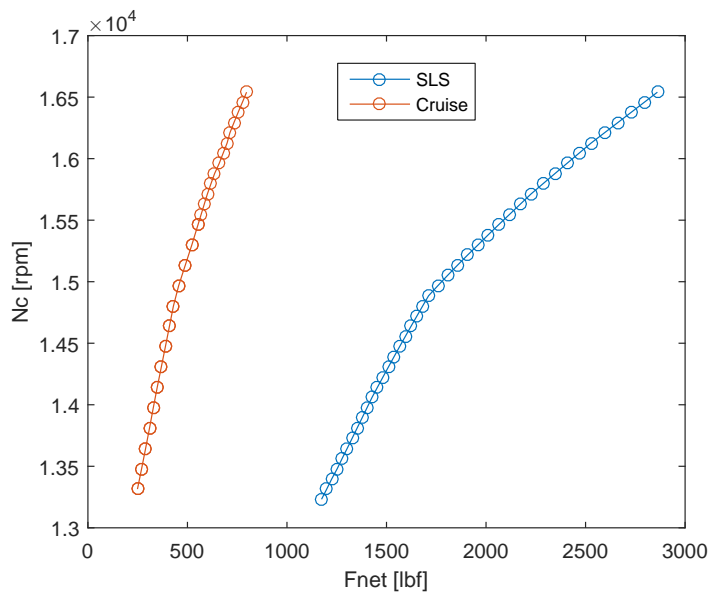

Figure 18. $N c$ vs. $F_{n}$, idle to max power.

\section{Summary}

This paper reviewed equations and techniques useful when simulating gas turbine performance. Topics covered include the Brayton cycle, performance maps, map scaling, design point criteria, and off-design effects. Additionally, the paper discussed an example performance analysis, detailing how changes in environmental conditions and power level affect the state of the system and how certain parameters can be utilized for engine control purposes. All comparison and modeling techniques were demonstrated through the creation of a turbojet simulation based on the J85 engine platform. This is accomplished by using the Toolbox for the Modeling and Analysis of Thermodynamic Systems or T-MATS software package. Combining Simulink ${ }^{\circledR}$ with industry standard thermodynamic simulation techniques, T-MATS offers a fast and convenient platform for the creation of system level turbomachinery models with application dependent fidelity and, generally, faster than real time execution. Models generated in this paper where created with a large number of assumptions and a less than ideal amount of data, and therefore are meant to be used only for student reference or educational material preparation. 


\section{Appendix}

Table A1. Cycle design data from literature and engine limits.

\begin{tabular}{|c|c|c|}
\hline Parameter & Value & Method \\
\hline \multicolumn{3}{|l|}{ Input } \\
\hline$P t_{\text {input }}$ & 14.696 psia & standard day SLS \\
\hline$T t_{\text {input }}$ & $518.67 \mathrm{R}$ & standard day SLS \\
\hline \multicolumn{3}{|l|}{ Compressor } \\
\hline$E f f$ & 0.87 & derived \\
\hline$P R$ & 7 & design value \\
\hline \multicolumn{3}{|l|}{ Cooling Flow } \\
\hline$W_{\text {cool }}$ & $0.01 \mathrm{pps}$ & $\begin{array}{c}\text { derived as fraction of compressor flow } \\
\text { and applied to inlet of turbine }\end{array}$ \\
\hline \multicolumn{3}{|l|}{ Burner } \\
\hline$W f$ & $0.7867 \mathrm{pps}$ & set based on $S F C$ and thrust \\
\hline$L H V$ & $18,400 \mathrm{BTU} / \mathrm{lbm}$ & assumed \\
\hline$E f f$ & 0.98 & derived \\
\hline$d P$ & 0.05 & derived \\
\hline \multicolumn{3}{|l|}{ Turbine } \\
\hline$E f f$ & 0.85 & derived \\
\hline$P R$ & 2.663 & derived \\
\hline \multicolumn{3}{|l|}{ Duct } \\
\hline$d P$ & 0.1 & derived \\
\hline \multicolumn{3}{|l|}{ Nozzle } \\
\hline$W_{\text {total }}$ & $44.7867 \mathrm{pps}$ & $W_{\text {design }}+W f$ \\
\hline$C_{f g}$ & 0.98 & derived \\
\hline \multicolumn{3}{|l|}{ Shaft } \\
\hline $\mathrm{N}$ & $16,540 \mathrm{rpm}$ & design \\
\hline
\end{tabular}




\section{References}

${ }^{1}$ Walsh, P.P., and Fletcher, P., Gas Turbine Performance, $2^{\text {nd }}$ ed., Blackwell Science and ASME, Fairfield, NJ, 1998.

${ }^{2}$ Mattingly, J.D., Elements of Gas Turbine Propulsion, McGraw-Hill series in aeronautical and aerospace engineering, McGraw-Hill, 1996.

${ }^{3}$ Chapman, J.W., Lavelle, T.M., May, R.D., Litt, J.S., Guo, T.H., "Toolbox for the Modeling and Analysis of Thermodynamic Systems (T-MATS) User's Guide," NASA/TM-2014-216638, January, 2014.

4 "www.cantera.org," as of 3/9/2016.

${ }^{5}$ Chapman, J.W., Lavelle, T.M., May, R.D., Litt, J.S., Guo, T.H., "Propulsion System Simulation Using the Toolbox for the Modeling and Analysis of Thermodynamic Systems (T-MATS)," AIAA-2014-3929, 2014 AIAA Joint Propulsion Conference, Cleveland, OH, Jul 28-30, 2014.

${ }^{6}$ Chapman, J.W., Lavelle, T.M., Litt, J.S., Guo, T.H., "A Process for the Creation of T-MATS Propulsion System Models From NPSS Data," AIAA-2014-3931, 2014 AIAA Joint Propulsion Conference, Cleveland, OH, Jul 28-30, 2014.

${ }^{7}$ Zinnecker, A.M., Chapman, J.W., Lavelle, T.M., Litt, J.S., "Developement of a twin-spool turbofan engine simulation using the Toolbox for the Modeling and Analysis of Thermodynamic Systems(T-MATS)," AIAA-2014-3930, 2014 AIAA Joint Propulsion Conference, Cleveland, OH, Jul 28-30, 2014.

${ }^{8}$ Milner, E.J. and Wenzel, L.M., "Performance of a J85-13 Compressor With Clean and Distorted Inlet Flow," NASA TM X-3304, December, 1975.

${ }^{9}$ Daly, M., Gunston, B., Jane's Aero-Engines, Issue 30, Jane's Information Group, London, England, 2011.

${ }^{10}$ Ypma, T. J., "Historical Devleopement of the Newton-Raphson Method," 1995 Society for Industrial and Applied Mathematics, SIAM Review, Vol.37, No. 4, December, 1995, pp. 531-551.

${ }^{11}$ Cengel, Y.A., Introduction to Thermodynamics and Heat Transfer, McGraw-Hill series in mechanical engineering, Irwin/McGraw-Hill, 1997.

${ }^{12}$ Gilat, A., Subramaniam, V.,Numerical Methods for Engineers and Scientists, $2^{\text {nd }}$ ed., John Wiley \& Sons, Inc., 2011.

${ }^{13}$ Oates, G. C. (ed.), Aircraft Propulsion Systems Technology and Design, AIAA Education Series, AIAA, New York, 1988.

${ }^{14}$ Hill, P., Peterson, C., Mechanics and Thermodynamics of Propulsion, $2^{\text {nd }}$ ed., Pearson, 1992.

${ }^{15}$ McKinney, J.S., "Simulation of Turbofan Engine. Part I. Description of Method and Balancing Technique", AFAPL-TR67-125, Pt.1, Air Force Systems Command, Wright-Patterson Air Force Base, Ohio, 1967.

${ }^{16}$ Jackson, P., Munson, K., Peacock, L., Jane's All the World's Aircraft 2011-2012, Jane's Information Group, London, England, 2011. 